Research Article

\title{
Monitoring and Analysis of Ground Settlement Induced by Tunnelling with Slurry Pressure-Balanced Tunnel Boring Machine
}

\author{
Hyunku Park (D), Ju-Young Oh, Dohyung Kim, and Seokbue Chang \\ Civil Business Unit, Engineering and Construction Group, Samsung CઐT Corporation, Seoul, Republic of Korea \\ Correspondence should be addressed to Hyunku Park; bowstringnine@gmail.com
}

Received 7 December 2017; Accepted 22 March 2018; Published 12 April 2018

Academic Editor: Venu G. M. Annamdas

Copyright (c) 2018 Hyunku Park et al. This is an open access article distributed under the Creative Commons Attribution License, which permits unrestricted use, distribution, and reproduction in any medium, provided the original work is properly cited.

\begin{abstract}
A case study of monitoring and analysis of ground settlement caused by tunnelling of stacked twin tunnels for underground metro line construction through the densely populated area using the slurry pressure-balanced TBM is presented. Detailed ground settlement monitoring was carried out for the initial stage of down-track tunnelling in order to estimate trough width factor and volume losses including face, shield, and tail losses. In addition, using the gap model, prediction of volume loss and ground settlement was carried out with consideration of the ground condition, TBM configurations, and actual operation data. The predictions of the gap model were compared with the observed results, and adjustment factors were determined for volume loss estimation. The adjusted factors were applied to predict ground settlement of the up-track tunnel, and its results were compared with the field measurements.
\end{abstract}

\section{Introduction}

The closed-type shield tunnel boring machine (TBM) has been widely applied to the tunnelling works in urban area to minimize impact of tunnel construction on the adjacent facilities and buildings. Compared to the conventional drill and blast tunnelling method, the shield TBM tunnelling has several advantages in the control of ground settlement [1]. First, the TBM excavates ground using a disc cutter or cutter bits mounted on the rigid steel cutterhead and imposes earth pressure or slurry pressure on the tunnel face to prevent squeezing movement of ground towards the tunnel face. After finishing excavation at the tunnel face, the shield TBM radially supports the inside of the excavated cavity based on the reaction from the thick steel shield skin plate. Then, concrete segment lining is installed just rear of the shield skin plate as a permanent ground supporting structure, where simultaneous backfilling of pressurized grout is also conducted at the annulus gap around the segment lining to restrict excessive radial contraction of the excavated ground. According to the field observations in the literatures [2-4], ground settlement during shield TBM tunnelling develops in the following steps in accordance with the abovementioned ground supporting mechanisms: (1) before and during tunnel face excavation, (2) during passage of the shield skin plate, and (3) after installation of segmental lining and backfill grouting. In general, the ground settlement in tunnelling is thought to be caused by ground loss, which is defined as a difference between actual and theoretical excavation volume. The ground loss develops due to internal deformation of the tunnel during excavation, which makes the actual amount of excavation to be larger than the theoretical amount. Hence, for TBM tunnelling, ground loss can be divided into three categories to be consistent with the abovementioned ground settlement steps as called face loss, shield loss, and tail loss [5]. The face loss is the ground loss caused by deformation of ground into tunnel face, and the shield loss is induced by the radial contraction of ground along the annular gap around the shield skin plate. The tail loss occurs along the annular void between ground and concrete segmental lining as a result of shrinkage or compression of backfill grout material. Because the closed-type shield TBM has measures for reducing the ground deformation at the tunnel face, around the shield skin plate and 
tail void, each ground losses can be estimated based on both the ground and tunnel operation conditions including TBM dimensions, face pressure, and backfill grouting pressure. For quantitative estimation of ground loss during shield TBM tunnelling, Lee et al. [5] proposed a gap model. The model estimates total ground loss as a linear sum of face loss, shield loss, and tail loss, where the each ground loss can be calculated based on simple elastic equations for the squeezing of tunnel face and the contraction of excavated cavity. However, the gap model has some limitations and uncertainties for practical use such as assumptions of isotropic stress condition of ground, friction between ground and shield skin plate, flow of bentonite slurry or backfill grout around shield, and shrinkage rate in backfill grout at tail void, which can be changed and affected by actual TBM operation conditions. Accordingly, the gap model has not been applied in the analysis of the observed ground settlement during TBM tunnelling. Instead, many researchers tried to correlate the volume loss with TBM operation data directly. However, because the process around shield TBM tunnelling is very complicated, most of the studies could not provide statistically meaningful relationships between the volume loss and operation parameters [2-4, 6, 7].

In practice, TBM tunnelling-induced ground settlement is mostly estimated based on the empirical relationships or guidelines. Those mostly provide site-specific or geologically specific characteristic parameters for prediction of ground settlement in terms of volume loss, that is, ground loss per unit area of tunnel excavation, and trough width, that is, distance from the tunnel centerline to the inflection point of the ground settlement trough [8-11]. However, the empirical methods are unable to consider effect of the supporting actions of the shield TBM in the calculation of volume loss, and consequently, the relatively conservative value of volume loss is usually adopted in ground settlement control. This will also lead conservative estimation of ground settlement, which may require unnecessary ground improvement or reinforcement as countermeasures. Hence, for economic design and planning of shield TBM tunnelling, reasonable prediction of volume loss based on reliable analysis of monitored ground settlement is essential.

This paper presents the results of monitoring and analysis of ground settlement induced by tunnelling of stacked twin tubes (down- and up-track tubes) for underground metro line construction through the densely populated area using the slurry pressure-balanced (SPB) TBM. For the initial stage of down-track tunnelling, comprehensive ground settlement monitoring was carried out in both transverse and longitudinal sections against the tunnelling direction to figure out the trough width factor and volume losses including face, shield, and tail losses. Besides, based on the ground condition, TBM configuration, and actual operation data, volume loss and ground settlement were predicted using the gap model. Then, the gap model's predictions were compared with the observations and adjustment parameters for total volume loss estimation, that is, linear summation of face, shield, and tail loss was introduced. At last, the adjusted gap model was applied to predict the ground settlement of up-track tunnelling and compared with the monitored results.

\section{Details of Shield TBM Tunnelling}

2.1. Tunnel Alignment and Ground Condition. The stacked twin TBM tunnels were constructed in shallow depth by crossing the densely populated area, and hence, it was required to minimize ground settlement and consequent damage to existing buildings. The tunnel alignment, adjacent condition of land occupation, and vertical geological profile are presented in Figure 1. Except for the launching station and a few hundred meters of the initial driving section, most of tunnel excavation was conducted below the heavy traffic roads with major transportation services. For TBM tunnel section 1, springlines of both the up- and down-track tunnels range from $10 \mathrm{~m}$ to $22 \mathrm{~m}$ below ground surface, which means most of the tunnel excavation was carried out in low ground cover condition. On the other hand, TBM tunnel section 2 shows the sufficient ground cover, where the TBM mostly passed below mountainous area with increased rock-head level.

Ground condition could be characterized as three strata from ground level as follows: reclaimed fill layer; alluvial layer; and decomposed granite rock layer. Table 1 summarizes the geotechnical parameters of the ground at tunnel depth. Fill layer is loose silt and sand, and alluvium layer is gravelly medium silty sand with high clayey contents. Depending on the degree of weathering, the decomposed granite layer was classified into four categories as moderately (MDG), highly (HDG), completely decomposed granite (CDG), and partially existing corestones, where MDG, HDG, and CDG could be classified very hard to hard rock, highly fractured normal to weak rock, and very weak rock to weathered soil, respectively. As can be seen from the longitudinal profile, the TBMs mostly drove through fill, alluvium, and CDG/HDG layers except for mountainous area, where the tunnel passes the MDG layer. Due to severe variation of rock-head level, mixed face conditions, that is, ground condition that soil and rock coexist within a tunnel face, frequently appeared along tunnel alignment which was composed of the alluvial layer and CDG/HDG layer or CDG/HDG layer and corestone layer. Groundwater level locates one to three meters below ground level, and hence, maximum three bar of groundwater pressure was expected at tunnel face.

\subsection{TBM Specification and Instrumentation. Aforementioned} ground conditions imply that there were unfavourable conditions for ground settlement control in TBM tunnelling. The mixed face conditions at the tunnel face can cause over excavation, and it will be resulted in additional ground settlement. In addition, severe groundwater inflow during excavation can be occurred due to high groundwater pressure and severe variation of permeability of ground around tunnel depth. Moreover, the shallow ground cover for up-track tunnel at the initial part of TBM tunnel section 1 was considered as most problematic. Besides, the CDG and HDG were found to be abrasive material for TBM excavation because the rocks have high content of quartz. In order to hedge the aforementioned unfavourable conditions for tunnelling, mixshield-type slurry pressure-balanced (SPB) TBMs were applied for both up- and 


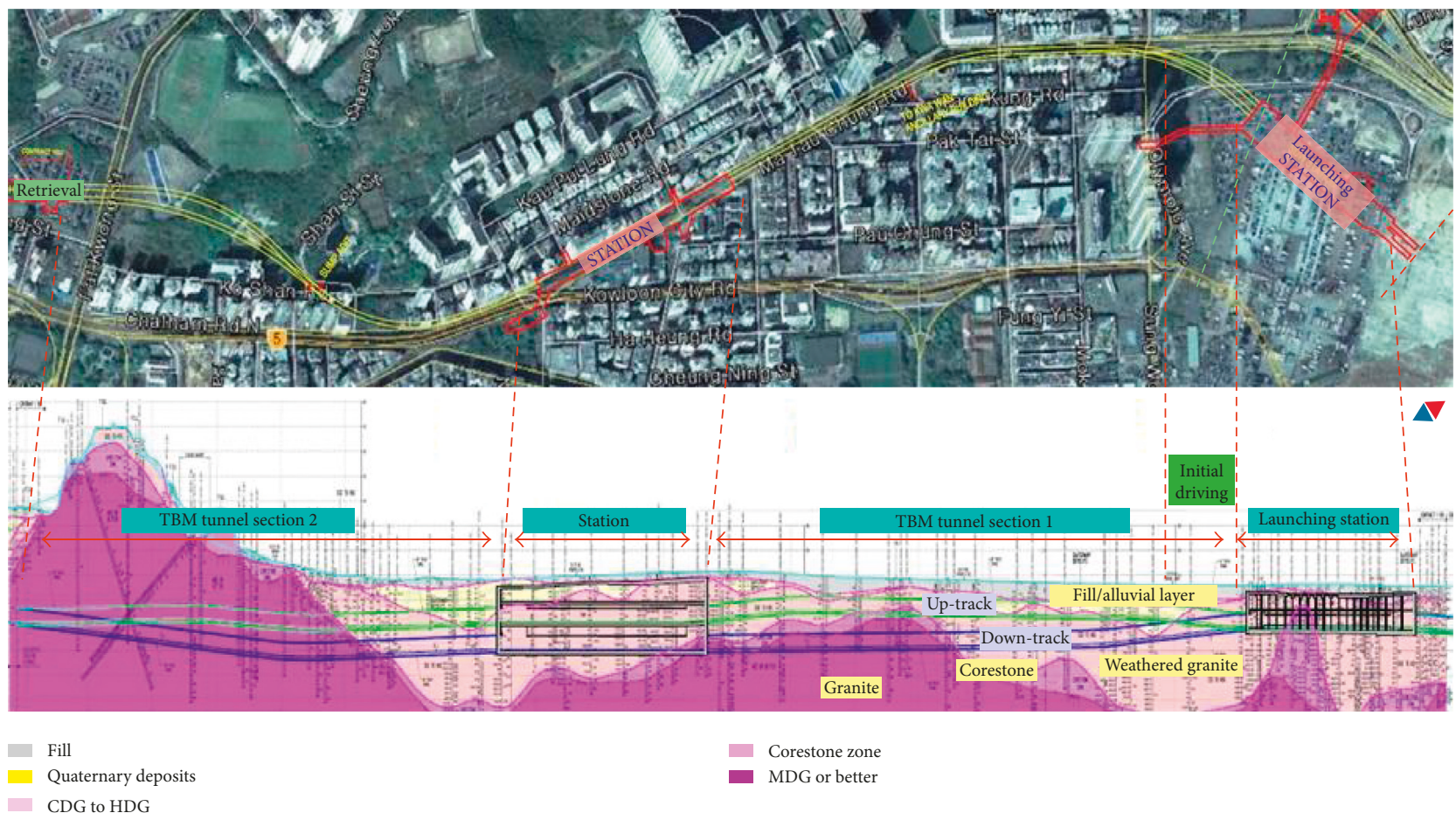

Figure 1: Tunnel alignment and geological profile.

TABLE 1: Geotechnical design parameters of the ground.

\begin{tabular}{|c|c|c|c|c|c|c|c|}
\hline $\begin{array}{l}\text { Ground } \\
\text { type }\end{array}$ & $\begin{array}{l}\text { Unit weight } \\
\left(\mathrm{kN} / \mathrm{m}^{3}\right)\end{array}$ & $\begin{array}{c}N_{\mathrm{SPT}} \\
\text { (down-track) }\end{array}$ & $\begin{array}{c}N_{\mathrm{SPT}} \\
\text { (up-track) }\end{array}$ & $\begin{array}{c}\text { Young's } \\
\text { modulus (MPa) }\end{array}$ & $\begin{array}{l}\text { Friction } \\
\text { angle }\left({ }^{\circ}\right)\end{array}$ & $K_{\mathrm{o}}$ & $\begin{array}{c}\text { Permeability, } \\
k(\mathrm{~m} / \mathrm{sec})\end{array}$ \\
\hline Fill & 19 & $<10$ & $<10$ & & 35 & 0.4 & $1 \times 10^{-7}$ to $4 \times 10^{-4}$ \\
\hline Alluvium & 19 & $30 \sim 35$ & $24 \sim 30$ & $1.5 \times N_{\mathrm{SPT}}$ & 35 & 0.4 & $1 \times 10^{-8}$ to $4 \times 10^{-5}$ \\
\hline CDG & 19 & $40 \sim 60$ & $35 \sim 41$ & & 35 & 0.4 & $1 \times 10^{-7}$ to $4 \times 10^{-5}$ \\
\hline HDG & 19 & \multicolumn{2}{|c|}{200} & 300 & 40 & 0.4 & \\
\hline MDG & 22 & \multicolumn{2}{|c|}{$\mathrm{UCS}=30 \sim 200 \mathrm{MPa}^{*}$} & $>1,000$ & 45 & 0.4 & $1 \times 10^{-7}$ \\
\hline
\end{tabular}

${ }^{*}$ UCS: unconfined compressive strength.

TABLE 2: Main specification of the TBM.

\begin{tabular}{lc}
\hline Item & Value \\
\hline Excavation diameter (including overcut) (mm) & 7,450 \\
Skin plate length (mm) & 11,450 \\
Skin plate diameter (tail) (mm) & 7,400 \\
Segment inner/outer diameter (mm) & $6,500 / 7,100$ \\
Maximum machine thrust (approximate) (kN) & 47,000 \\
Maximum torque (approximate) (kNm) & 5,000 \\
Operating slurry pressure (bar) & 4.0 \\
Backfill grout & Bicomponent simultaneous injection \\
\hline
\end{tabular}

down-track tunnels. In general, compared to earth pressure-balanced (EPB) TBM, SPB TBM is more compatible with both rock and soft ground and has advantages in reducing not only the groundwater inflow in permeable or fractured ground but also the abrasion of the cutting tool based on the flow of bentonite slurry around the cutterhead. Table 2 presents the main specifications of the tunnel boring machines.

Because most of the tunnel alignment was along the heavy traffic road crossing the dense commercial area, it was hard to measure the ground settlement close to the tunnel. Hence, in this study, initial $300 \mathrm{~m}$ section from the launching station, where the public park located, was chosen for installation of settlement monitoring arrays and analysis of ground settlement behavior. The longitudinal profile of the monitoring section is presented in Figure 2, where both the down- and up-track tunnels were driven with low ground cover through the fill, alluvium, and upper part of CDG layers, which are thought to be susceptible to settlement. In addition, both of the tunnels were classified as shallow 


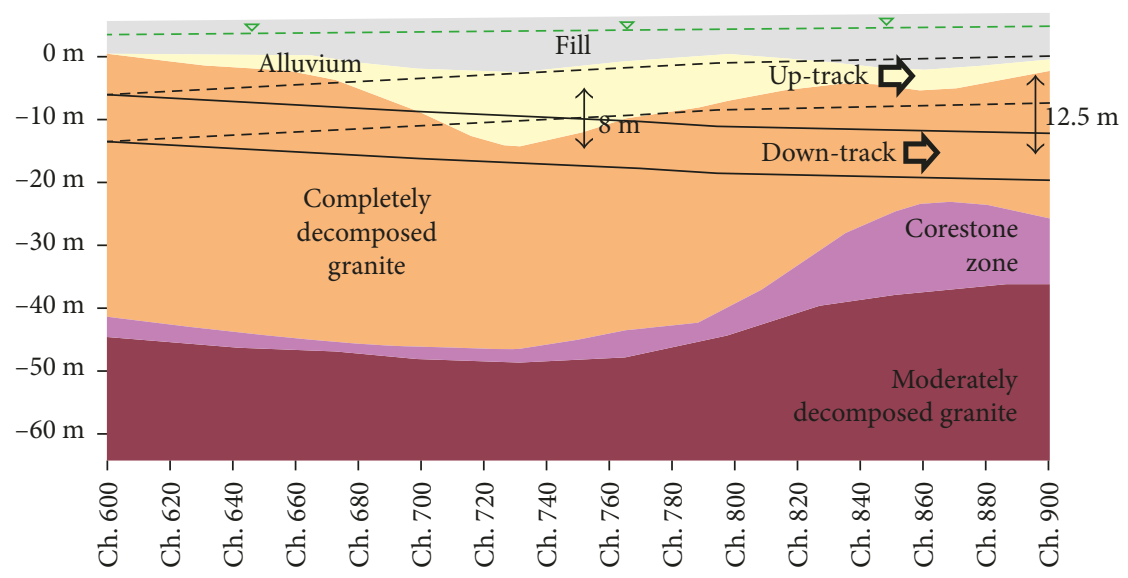

FIGURE 2: Longitudinal profile of the monitoring section.
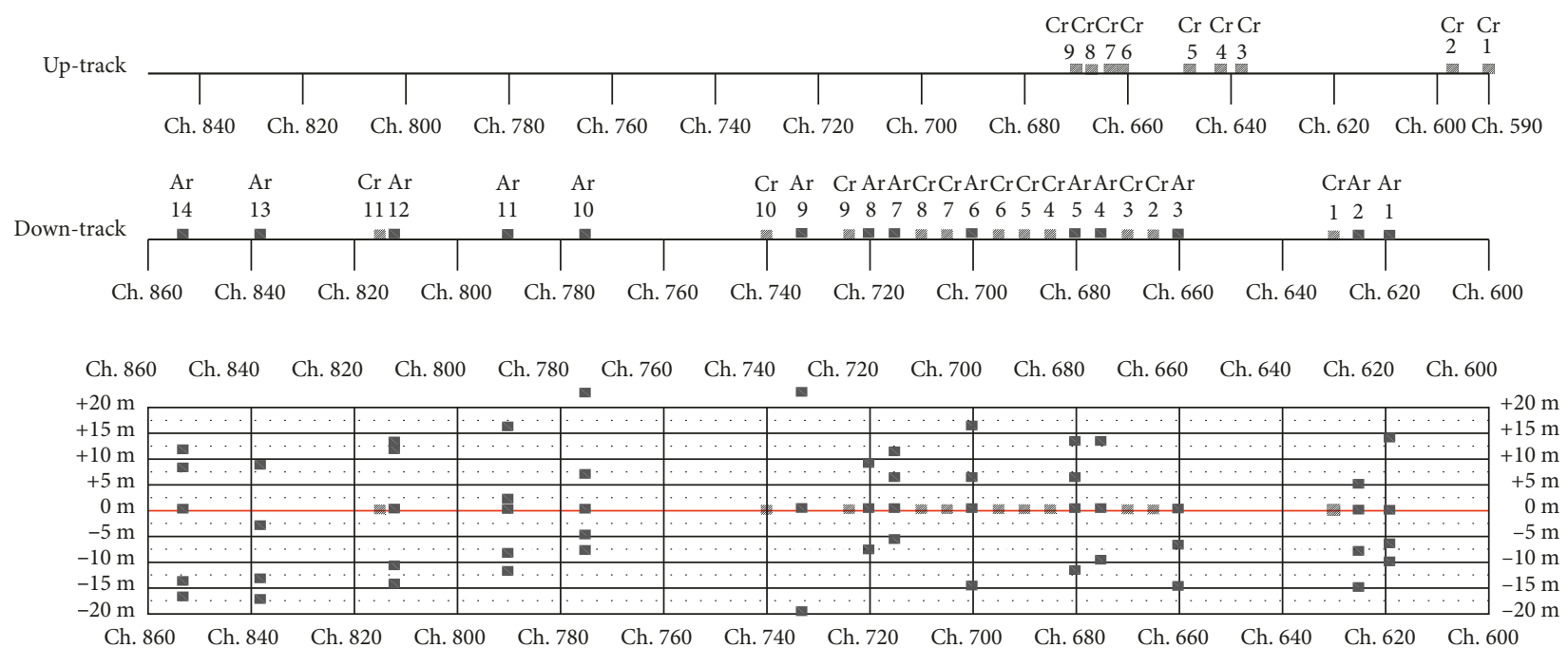

FiguRE 3: Instrumentation for ground settlement measurement.

tunnels because cover depth of up-track and down-track tunnels is less than twice of the excavation diameter, and in the case of the up-track tunnel, cover depth was occasionally less than the excavation diameter.

Figure 3 shows the plan views for the locations of ground settlement measurement points with reference to tunnel alignment. Because the down-track tunnelling was planned to start several months earlier than the up-track tunnel excavation, a detailed instrumentation layout was adopted to the down-track tunnel in order to characterize ground settlement behavior and volume loss development. The transverse settlement troughs were measured from fourteen settlement arrays. In addition, to monitor sequentially developing settlement at each stage of TBM advance as face excavation, passage of the shield skin plate, segment ring build with tail void grouting, eleven crown settlement points were instrumented directly above the tunnel centerline. Meanwhile, monitoring of only a few ground settlement points was allowed for the up-track tunnel due to change of construction site utilization plan around the public park area after passing the TBM for the down-track tunnel. As a result, along initial $100 \mathrm{~m}$ section of the up-track tunnel, only 9 ground settlement points could be installed above tunnel crown.

Figure 4 depicts the monitored face and backfill pressure applied in tunnelling for both up- and down-track tunnelling. In order to prevent ground heaving during the up-track tunnel due to very shallow cover depth, actual face pressure was controlled less than $150 \mathrm{kPa}$. In the down-track case, low face pressure was maintained for the initial driving stage and then gradually increased with cover depth increase. On the other hand, $400 \mathrm{kPa}$ of pressure was consistently applied for backfill grouting to minimize ground settlement.

\section{Analysis Methods}

3.1. Ground Settlement Prediction Using Gap Model. For the theoretical evaluation of the volume loss with consideration of ground characteristics, TBM's dimensions, and actual TBM operation conditions, the modified gap model by Loganathan [12] was applied in this study. Firstly, the model calculates the face loss $V_{\mathrm{f}}$ from the following equation: 


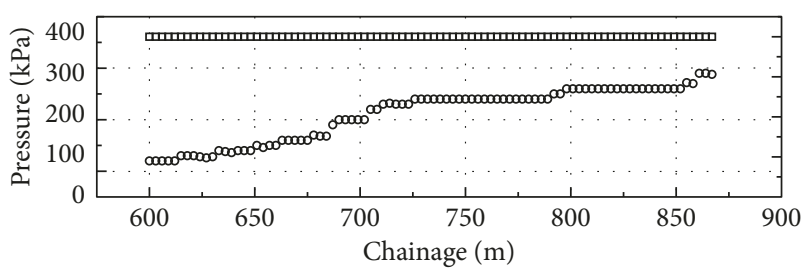

- Face pressure

- Backfill pressure

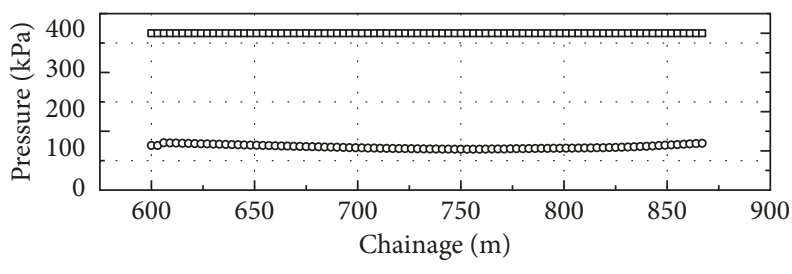

- Face pressure

- Backfill pressure

(a)

(b)

FIGURE 4: Face pressure and backfill pressure for tunnelling: down-track (a) and up-tack (b) tunnel.

$$
\begin{aligned}
& V_{\mathrm{f}}=\frac{a b P_{0} / E_{\mathrm{u}}}{r} \times 100(\%), \\
& \text { 0.7, } \quad q_{\mathrm{u}}>100 \mathrm{kPa} \\
& a=0.9,25 \mathrm{kPa}<q_{\mathrm{u}}<100 \mathrm{kPa} \text {, } \\
& \text { 1.0, } \quad q_{\mathrm{u}}<25 \mathrm{kPa} \\
& \text { 1.12, } \quad N_{\mathrm{R}}<3 \\
& b=0.63 N_{\mathrm{R}}-0.77,3<N_{\mathrm{R}}<5 \text {, } \\
& 1.07 N_{\mathrm{R}}-2.55, \quad N_{\mathrm{R}}>5
\end{aligned}
$$

where $a$ is the coefficient for the frictional resistance between the ground and the TBM shield skin plate as determined from (2), $b$ is the coefficient accounting for the squeezing movement of ground at tunnel face as depicted in (3), $P_{0}$ is the net stress reduction at the tunnel axis, $E_{\mathrm{u}}$ is undrained Young's modulus value of ground at tunnel axis depth, and $r$ is the excavation diameter of the TBM.

In (2) and (3), $q_{\mathrm{u}}$ is the unconfined uniaxial strength, $N_{\mathrm{R}}$ is the stability number defined as follows:

$$
N_{\mathrm{R}}=\frac{\left(\gamma H-P_{\mathrm{i}}\right)}{C_{\mathrm{u}}},
$$

where $\gamma$ is the total unit weight of ground, $H$ is the depth of the tunnel axis from ground level, $P_{\mathrm{i}}$ is the face pressure, and $C_{u}$ is the undrained shear strength of ground at tunnel axis.

Then, the model estimates the shield loss $V_{\text {sh }}$ from (5) by considering the radial contraction $\left(U_{s}\right)$ of ground around shield plate. The contraction $U_{s}$ is determined as a half of the elastic deformation of the excavated cavity as expressed in (6). The contraction $U_{s}$ cannot be larger than the shield gap $g_{\text {sh }}$ due to the existence of the shield skin plate, where the shield gap is defined as a summation of overcut thickness $t_{\mathrm{b}}$ and shield taper amount $t_{\mathrm{t}}$ :

$$
\begin{aligned}
V_{\mathrm{sh}} & =\frac{0.5 \times U_{\mathrm{s}}}{r} \times 100(\%), \\
U_{\mathrm{s}} & =\frac{r \times(1+\nu) \times\left(\sigma_{\mathrm{v}}-P_{1}\right)}{E_{\mathrm{u}}} \leq g_{\mathrm{sh}}=t_{\mathrm{b}}+t_{\mathrm{t}},
\end{aligned}
$$

where $v$ is Poisson's ratio, $\sigma_{\mathrm{v}}$ is the vertical total stress at tunnel springline, and $P_{1}$ is the resisting pressure around shield skin plate
Tail loss $V_{\mathrm{t}}$ can be estimated from (7), which assumes $10 \%$ radial shrinkage of backfill grout during its curing after injection into tail void having thickness $t_{\mathrm{v}}$ :

$$
V_{\mathrm{t}}=\frac{0.1 \times t_{\mathrm{v}}}{r} \times 100(\%) \text {. }
$$

Then, the total volume loss $V_{\mathrm{L}}$ is defined as a linear summation of face, shield, and tail losses as depicted in the following equation:

$$
V_{\mathrm{L}}=V_{\mathrm{f}}+V_{\mathrm{sh}}+V_{\mathrm{t}}(\%)
$$

Based on the Gaussian distribution approximation [13] as depicted in the following equation, tunnelling-induced ground settlement trough in the transverse section can be estimated:

$$
S(x)=\frac{V_{\mathrm{L}} A_{\mathrm{t}}}{\sqrt{2 \pi i_{x}}} \times \exp \left(-\frac{x^{2}}{2 i_{x}^{2}}\right),
$$

where $V_{\mathrm{L}}$ is the total volume loss, $i_{x}$ is the trough width defined as the lateral distance of inflection point of the settlement trough from the tunnel axis, $x$ is the lateral distance from the tunnel axis, and $A_{\mathrm{t}}$ is the tunnel cross-sectional area.

3.2. Estimation of Volume Loss from Monitoring Results. As mentioned above, the transversal ground settlement trough induced by tunnelling denotes the Gaussian distribution. Hence, on the basis of the Gaussian curve fitting based on the least square method for the settlement trough, the total volume loss $V_{\mathrm{L}}$ and trough width $i_{x}$ can be determined. On the other hand, for final ground settlement measured at directly above tunnel crown, total volume loss can be estimated as depicted in the following equation:

$$
V_{\mathrm{L}}=\frac{\sqrt{2 \pi i_{x}} \times S_{\max }}{A_{\mathrm{t}}} \times 100(\%),
$$

where $S_{\max }$ is the maximum ground settlement developed above the tunnel axis $(x=0)$.

If ground settlement at each stage of TBM advance, $\Delta S_{\mathrm{v}}$, as face excavation, passage of the shield skin plate, and ring build and tail grouting can be individually separated from the measurement, volume loss at each stage $\Delta V_{\mathrm{L}}$ can be calculated using the following equation: 


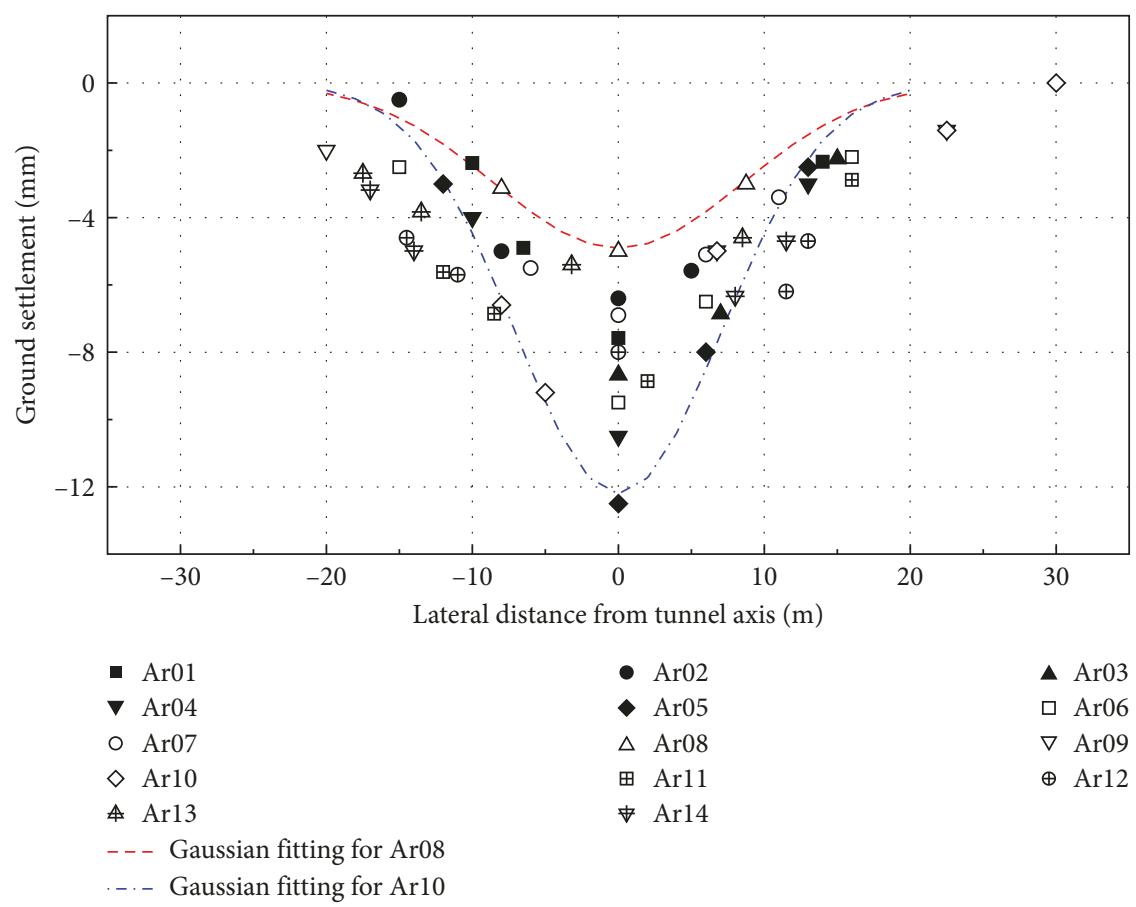

Figure 5: Transversal settlement troughs measured in down-track tunnelling.

TABLE 3: Estimated volume loss parameters from down-track tunnelling.

\begin{tabular}{|c|c|c|c|c|c|c|c|c|c|c|c|c|c|c|c|}
\hline \multirow{2}{*}{ Parameter } & \multirow{2}{*}{ Average } & \multicolumn{14}{|c|}{ Settlement arrays } \\
\hline & & 1 & 2 & 3 & 4 & 5 & 6 & 7 & 8 & 9 & 10 & 11 & 12 & 13 & 14 \\
\hline$S_{\max }(\mathrm{mm})$ & -8.1 & -7.3 & -6.8 & -9.1 & -10 & -12 & -8.9 & -6.7 & -4.9 & -6.3 & -11 & -9.1 & -8 & -5.5 & -7.4 \\
\hline$V_{\mathrm{L}}(\%)$ & 0.44 & 0.32 & 0.3 & 0.44 & 0.46 & 0.49 & 0.47 & 0.35 & 0.24 & 0.42 & 0.43 & 0.59 & 0.63 & 0.47 & 0.58 \\
\hline$i_{x} / H$ & 0.52 & 0.48 & 0.49 & 0.5 & 0.44 & 0.41 & 0.5 & 0.49 & 0.46 & 0.67 & 0.35 & 0.54 & 0.63 & 0.66 & 0.61 \\
\hline
\end{tabular}

$$
\Delta V_{\mathrm{L}}=\frac{\sqrt{2 \pi i_{x}} \times \Delta S_{\mathrm{v}}}{A_{\mathrm{t}}} \times 100(\%) .
$$

\section{Results and Discussion}

4.1. Results of Monitoring and Analysis for Down-Track Tunnel. Figure 5 shows the transversal settlement troughs measured from the settlement point arrays installed along the down-track tunnel. In common, the settlement troughs were developed in the form of the Gaussian distribution, where the maximum ground settlement at the tunnel axis $(x=0)$ ranges from 5 to $13 \mathrm{~mm}$, and the width of the trough is less than $30 \mathrm{~m}$.

Based on the Gaussian curve fitting of the monitored transverse settlement troughs, total volume loss $V_{\mathrm{L}}$ and trough width factor $k\left(=i_{x} / H\right)$ were evaluated as summarized in Table 3. The back-calculated total volume loss and trough width factor range from $0.3 \%$ to $0.63 \%$ and from 0.35 to 0.66 , where their averages are $0.43 \%$ and $0.52 \%$, respectively. Those are similar to the values reported regarding tunnelling-induced volume loss in weathered granite ground around this site [14].

Figure 6 illustrates the variation of ground settlement with the elapsed time after TBM face arrival on the crown settlement monitoring point, where settlement development can be divided into four stages. First, small preceding ground heaving occurred in advance to arrival of the TBM face, which might be caused by face pressure. Then, immediate settlement developed as TBM face excavation initiated on day zero. When the shield skin plate passed below the monitoring point in 1 2 days, settlement rapidly increased, and finally, there were gradual increases of ground settlement after ring build for several tens of days, which finally converged to the constant value after several tens of days.

For each curves in Figure 6, logistic curve fittings were carried out to approximate the measured settlement and to estimate the relative ratio of settlement induced at each stage of the TBM drive against total settlement. From Figure 7, it is found that, on average, $6 \%, 30 \%$, and $64 \%$ of final settlement were developed during face excavation, skin plate passage, and ring build, respectively. This result implies that, for the slurry pressure-balanced TBM, major ground settlement develops during shield advance and ring build period while minor settlement is induced in face excavation.

The total volume loss evaluated from the field monitoring of the down-track tunnel was compared with the gap model's prediction as presented in Figure 8(a). It is found that, in the most of monitoring area, the estimation of the 


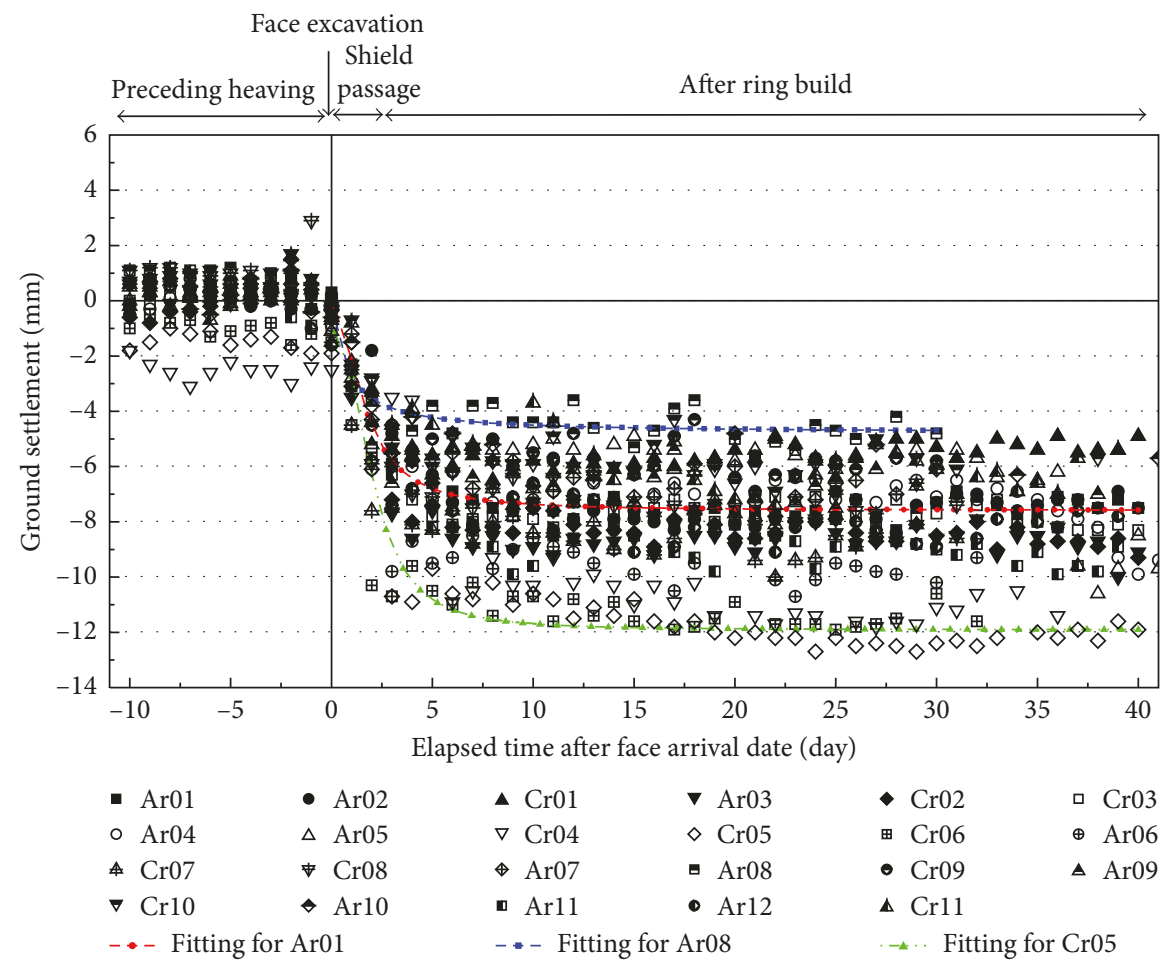

FIgURE 6: Variation of settlement above the tunnel crown with TBM drive sequences (down-track).

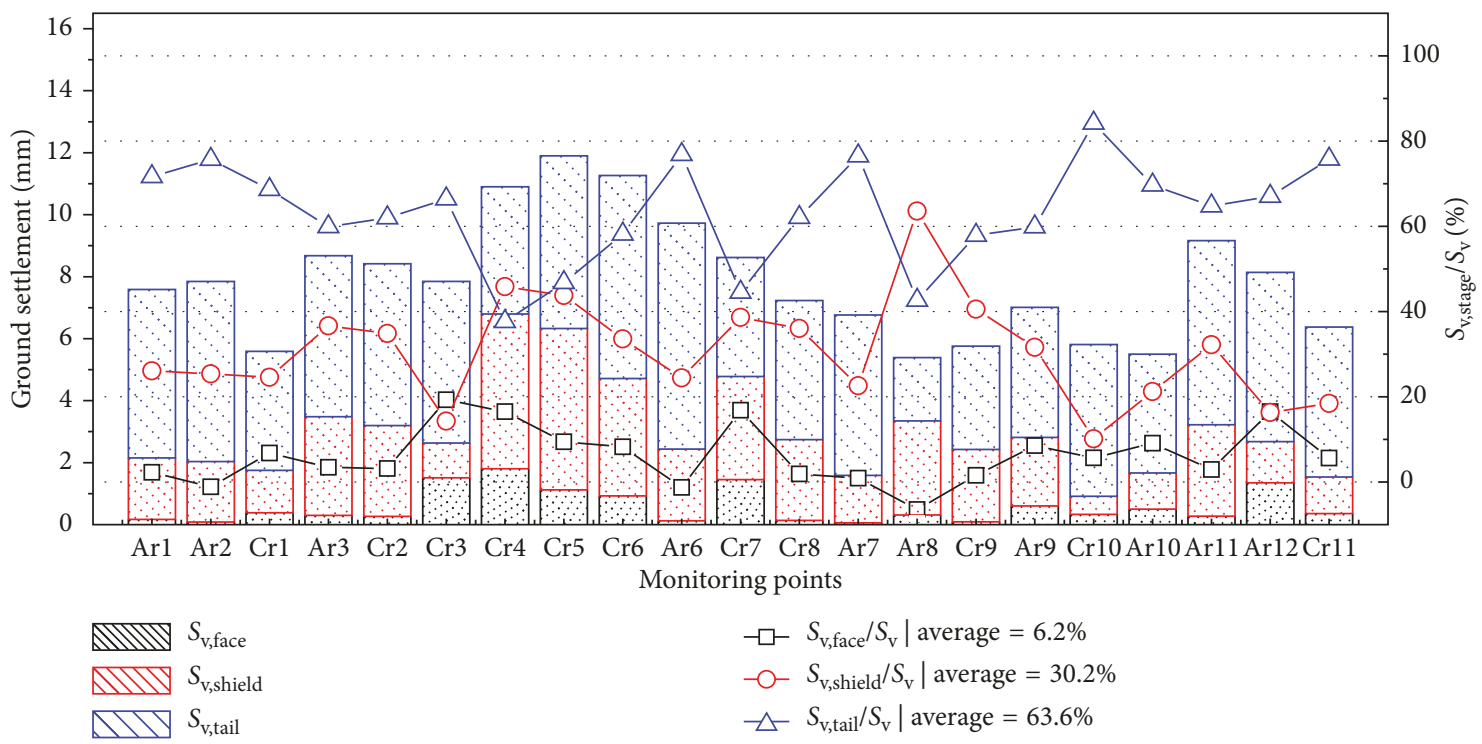

Figure 7: Settlement at face, shield, and tail and relative magnitude.

gap model is larger than the observed result. In particular, for the section where the TBM operation was stopped for preparation of the main drive, that is, from Chainage 670 to Chainage 700 , actually developed volume loss was much larger than the predictions. Figures 8(b) and 8(c) show the comparisons of the shield and tail loss between field monitoring and gap model, in which face loss was not considered to be compared because its magnitude is negligible. The gap model reasonably predicted the shield loss close to the measured values which mostly ranges within 0.05 to $0.15 \%$.
Especially, the gap model predicted very high large value of shield loss up to $0.23 \%$ at the region where face pressure was lowered to $100 \mathrm{kPa}$ for maintenance, of which observed volume loss was nearly $0.3 \%$. Meanwhile, as can be seen from Figure $8(\mathrm{c})$, the gap model mostly overestimated the tail loss compared to the measurement, where 0.4 to $0.45 \%$ of tail loss was predicted while less than $0.3 \%$ of tail loss was observed from field monitoring. On the other hand, or the region between Chainage 825 and Chainage 850 , nearly $0.5 \%$ of tail loss occurred, which is almost 1.2 times of the predicted value. 

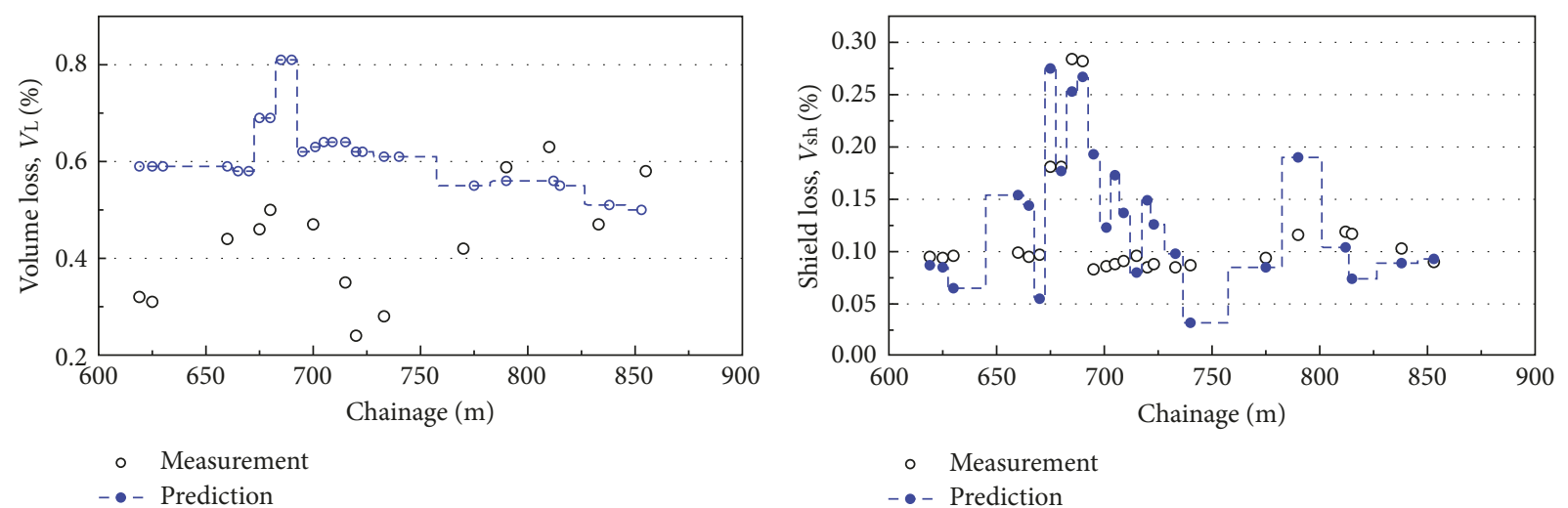

(a)
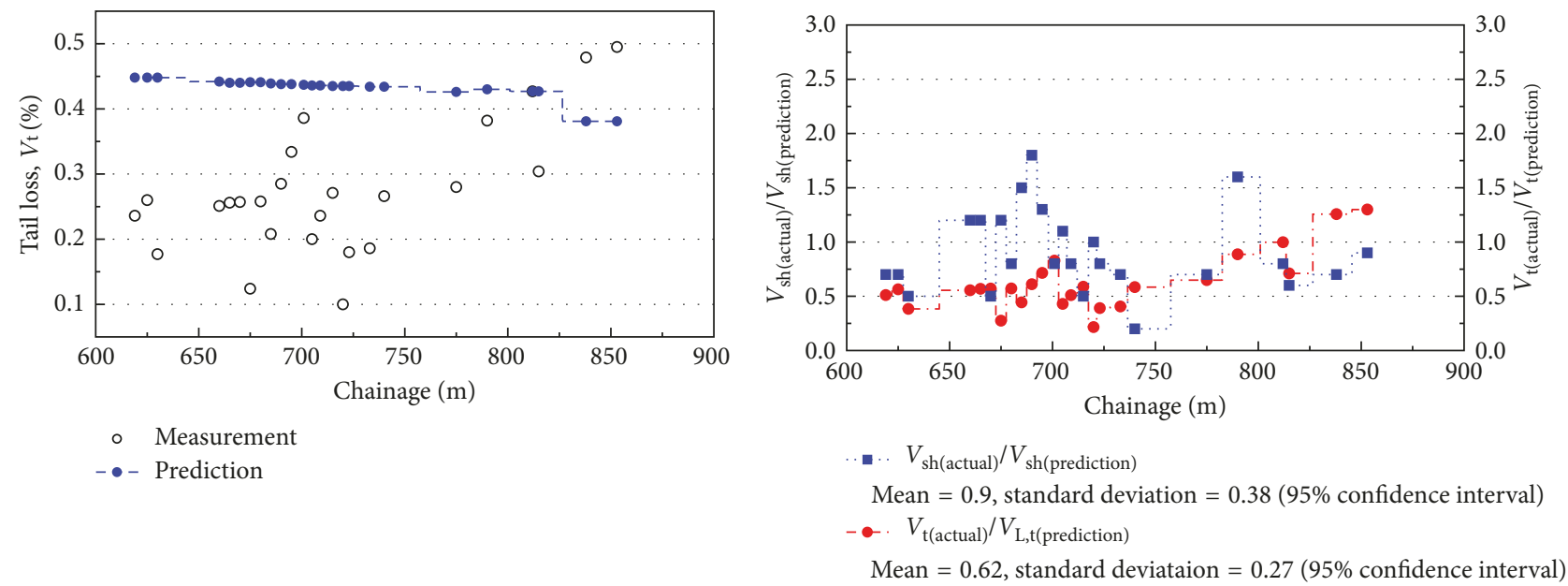

(c)

(d)

Figure 8: Comparison of volume loss between measurement and predictions for down-track tunnel: (a) total volume loss, (b) shield loss, (c) tail loss, and (d) relative magnitude of observed/predicted volume losses.

Figure $8(\mathrm{~d})$ summarizes the relative ratio of observed shield and tail losses with reference to the gap model estimations. Averagely, the back-calculated shield loss and tail loss are found to be $90 \%$ and $62 \%$ of the predictions of the gap model, of which the standard deviations are $38 \%$ and $27 \%$, respectively. These results imply that, for this site, volume loss development in the actual TBM drive can be predicted with the gap model by adopting adjusting coefficients for shield $\beta$ and tail loss $\gamma$ as depicted in (12), where $\beta$ and $\gamma$ range from $52 \%$ to $128 \%$ (mean: $90 \%$ ) and $35 \%$ to 99\% (mean: 62\%), respectively.

$$
V_{\mathrm{L}}=\alpha \times V_{\mathrm{f}}+\beta \times V_{\mathrm{sh}}+\gamma \times V_{\mathrm{t}}(\%) .
$$

4.2. Analysis of Up-Track Tunnel Monitoring Results. The variation of ground settlement observed during the up-track tunnel is plotted in Figure 9. Preceding settlement is found much before arrival of the TBM at the monitoring point as a resultant of down-track tunnelling. Similar to the downtrack tunnel case, settlement developed in four stages as minor ground heaving followed by settlement in face excavation, in shield passage, and after ring build.
By applying the adjusting factors for shield loss and tail loss estimation obtained from the down-track tunnel analysis, upper, medium, and lower values of settlement were estimated for the up-track tunnel case using the actual operation data recorded in the down-track TBM and trough width factor $\left(i_{x} / H\right)$ obtained from the Gaussian curve fitting for the down-track tunnel. The predictions were compared with the measurements as shown in Figure 10, where the preceding settlement caused by the up-track tunnel was excluded from the measurement. It is found that all the observed settlements were less than the predictions with medium and lower predictions except for the observations around Chainage 590 which is very close to the launching station. This result can be thought that the prediction with medium adjusting factors for shield and tail losses obtained from down-track analysis provided reasonable estimation of ground settlement in the up-track tunnel.

\section{Conclusion}

In this paper, a case study of volume loss estimation for the SPB TBM tunnelling case was carried out based on both monitoring and prediction. Tunnelling-induced ground 


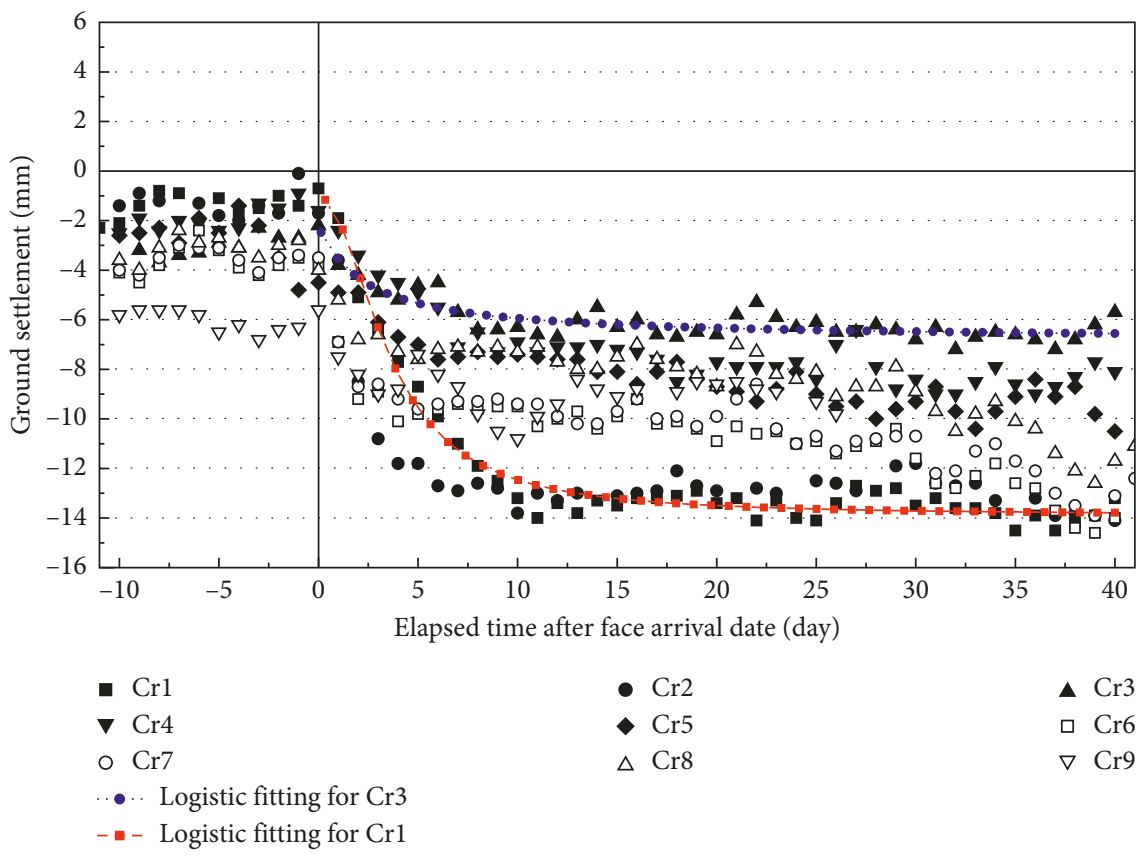

Figure 9: Variation of the settlement above tunnel crown with TBM drive sequences (up-track).

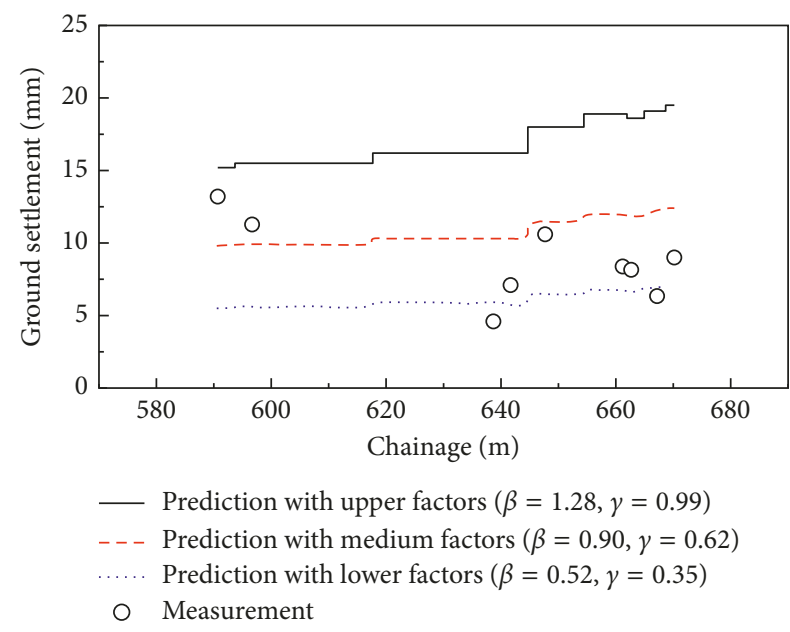

Figure 10: Comparison of ground settlement between measurement and predictions (up-track).

settlement was measured by using a series of settlement monitoring array. From the monitored later settlement troughs, total volume loss and trough factors were estimated by the Gaussian curve fitting, and the estimations were found to be coinciding with reported values. Monitoring results at each monitoring points along the tunnel axis indicated that settlement occurs in three stages as TBM advances: face excavation, shield passage, and ring build and tail grouting. In the case of the SPB TBM, most of ground settlement was occurred in shield advance and tail grouting, while negligible settlement was measured in face excavation. Besides, prediction of volume loss was conducted by adopting a gap model, in which TBM configurations, geotechnical condition of ground, and operation condition were taken into account for the calculation of face, shield, and tail losses. The predicted results were compared with the monitored volume loss. It was found that the gap model estimated shield loss reasonably and provided significantly larger evaluation of tail loss. By consideration of the difference between the observation and the estimation for the down-track, adjusting factors were determined for the gap model's predictions for shield and tail losses, and the factors were applied for prediction of ground settlement in the uptrack tunnel. The observed results were mostly within the predicted settlement range, where medium factors provided reliable estimation of ground settlement on average.

\section{Conflicts of Interest}

The authors declare that there are no conflicts of interest regarding the publication of this paper.

\section{Acknowledgments}

This research was supported by the Korea Agency for Infrastructure Technology Advancement under the Ministry of Land, Infrastructure and Transport of the Korean Government (Project no. 15SCIP-B066321-03).

\section{References}

[1] A. Bezuijen and K. J. Bakker, "Bentonite and grout flow around a TBM," in Proceedings of the WTC 2007, Prague, Czech Republic, 2011.

[2] V. Fargnoli, D. Boldini, and A. Amorosi, "TBM tunnellinginduced settlements in coarse-grained soils: the case of the new Milan underground line 5," Tunnelling and Underground Space Technology, vol. 38, pp. 336-347, 2013.

[3] B. D. Jones, "Low-volume-loss tunnelling for London ring main extension," Geotechnical Engineering, vol. 163, pp. 167-185, 2010.

[4] T. Sugiyama, T. Hagiwara, T. Nomoto et al., "Observations of ground movements during tunnel construction by slurry 
shield method at the Dockland Light Railway Lewisham Extension-East London," Soils and Foundations, vol. 39, no. 3, pp. 99-112, 1999.

[5] K. M. Lee, R. K. Rowe, and K. Y. Lo, "Subsidence owing to tunneling. I. Estimating the gap parameter," Canadian Geotechnical Journal, vol. 29, pp. 929-940, 1992.

[6] S. Suwansawat, Earth Pressure Balance (EPB) Shield Tunneling in Bangkok: Ground Response and Prediction of Surface Settlements Using Artificial Neural Networks, Ph.D. thesis, Department of Civil and Environmental Engineering, Massachusetts Institute of Technology, Cambridge, MA, USA, 2002.

[7] J. Wongsaroj, E. X. Borghi, K. Soga et al., "Effect of TBM driving parameters on ground surface movements: channel tunnel rail link contract 220," in Proceedings of the 5th International Symposium TC28, Amsterdam, Netherlands, June 2005.

[8] FHWA, Road Tunnel Manual (FHWA-NHI-09-010), Federal Highway Administration, Washington, DC, USA, 2009.

[9] LTA, Particular Specification, Thomson Line Contract T212, LTA, Singapore, 2013.

[10] R. J. Mair and R. N. Taylor, "Bored tunnelling in the urban environment," in Proceedings of the 14th International Society for Soil Mechanics and Geotechnical Engineering (ISSMGE), Hamburg, Germany, 1997.

[11] MRTC, Consultancy Agreement no. C1105-Shatin to Central Line, MRTC, Hong Kong, China, 2011.

[12] N. Loganathan, An Innovative Method for Assessing Tunnelling-Induced Risks to Adjacent Structures", Parsons Brinckerhoff, Inc., New York, NY, USA, 2011.

[13] R. B. Peck, "Deep excavations and tunneling in soft ground, state-of-art report," in Proceedings of the 7th International Conference on Soil Mechanics and Foundation Engineering (ISSMFE), Mexico City, Mexico, August 1969.

[14] R. A. Forth and C. B. B. Thorley, "Ground and building settlement due to tunnelling in Hong Kong," Land Subsidence, vol. 234, pp. 149-160, 1995. 


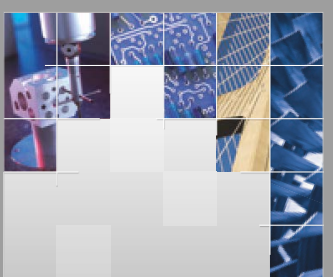

\section{Enfincering}
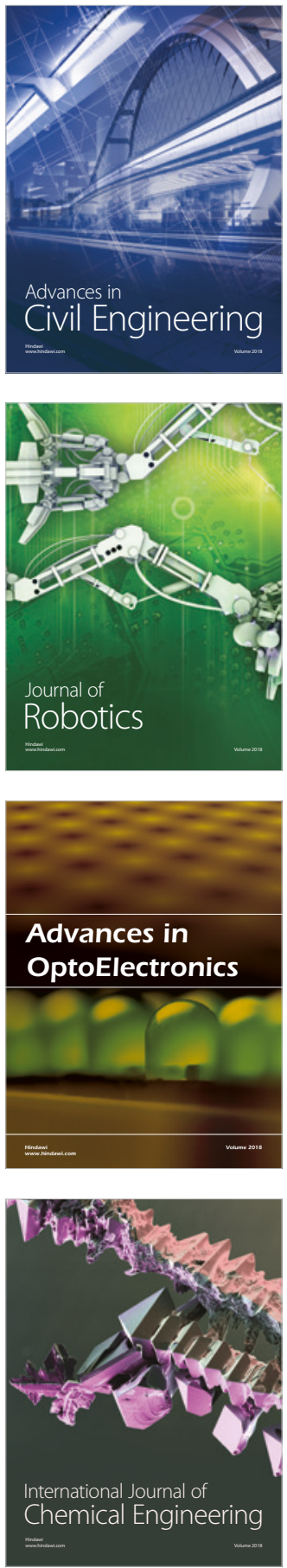

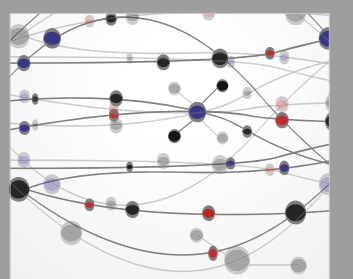

\section{Rotating \\ Machinery}

The Scientific World Journal

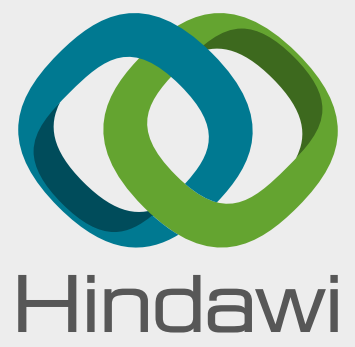

Submit your manuscripts at

www.hindawi.com
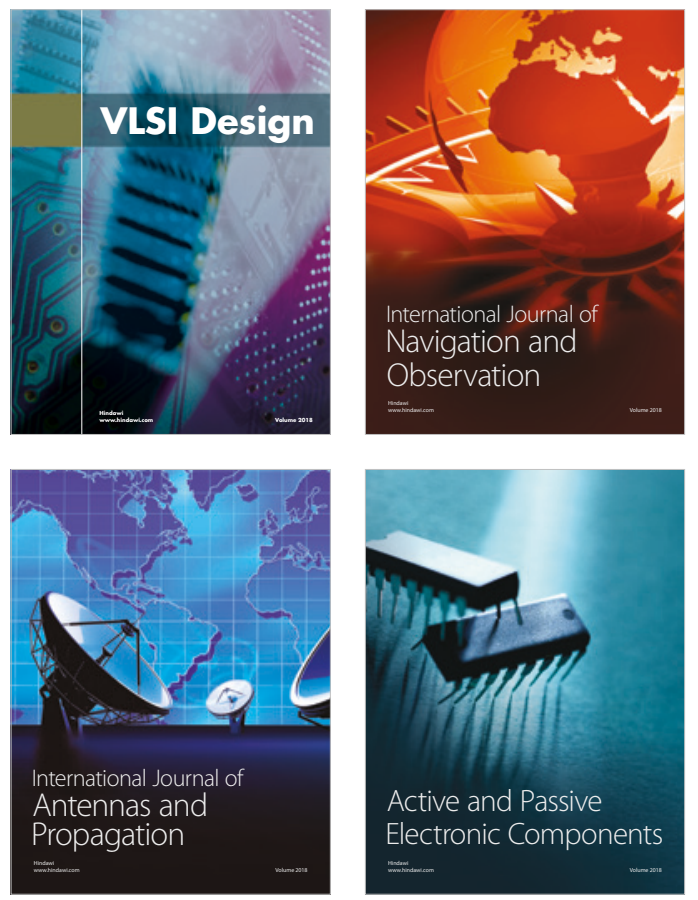
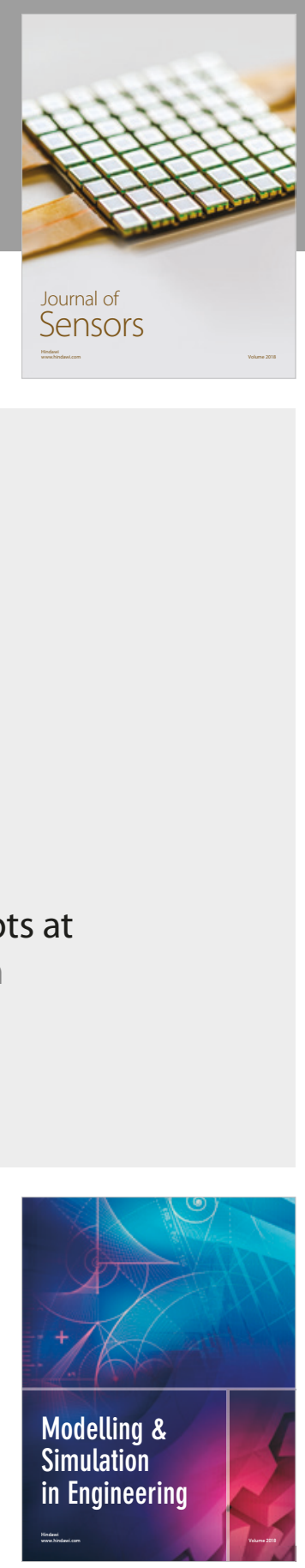

\section{Advances \\ Multimedia}
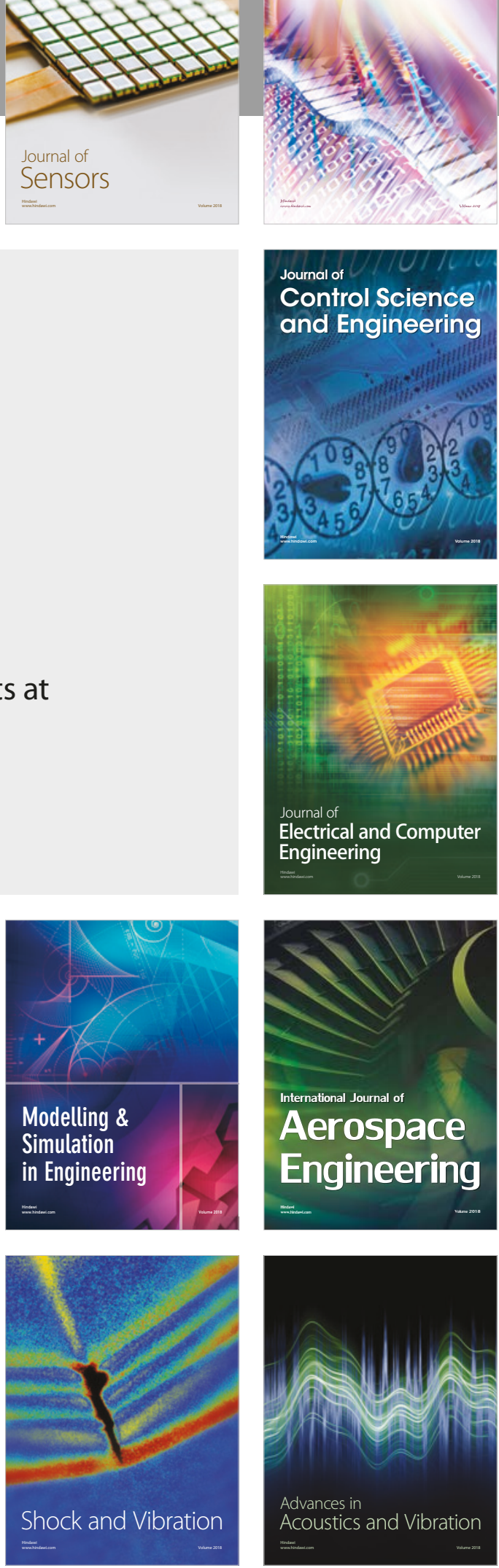